\title{
Impact of oxidative stress on semen parameters in normozoospermic infertile men: a case-control study
}

\author{
Ayad Palani ${ }^{* *}$ (1) and Ahmed Alahmar ${ }^{2}$ (1)
}

\begin{abstract}
Background: Oxidative stress has been implicated in male infertility through decrease in sperm quality. However, men with normal semen parameters (normozoospermia) may be unable to fertilize their partners even when they have normal sperm function. Thus, they would be considered infertile. The purpose of this study was to investigate the role of oxidative stress in the pathogenesis of unexplained male infertility.

Methods: In this case-control study, infertile men with normozoospermia $(n=46)$ and fertile control group $(n=21)$ underwent seminal fluid analyses according to WHO 2010 criteria. Serum and seminal plasma levels of total antioxidant capacity (TAC), glutathione, malondialdehyde, uric acid and albumin were also measured using colorimetric methods.

Results: The level of total antioxidant capacity in both serum and seminal plasma was significantly lower in normozoospermic infertile men in comparison with fertile group $(p<0.0001)$. However, no significant differences were observed in serum and seminal plasma levels of glutathione, uric acid, albumin and malondialdehyde between infertile and fertile groups.

Conclusion: LOW TAC level induces oxidative stress and consequently causes sperm dysfunction and male infertility. Estimation of TAC can be a useful tool in the diagnosis of male infertility. Antioxidant supplementation should be considered in the treatment of oxidative stress-induced male infertility.
\end{abstract}

Keywords: Unexplained infertility, Normozoospermia, Total antioxidant capacity, Antioxidants

\section{Background}

Medical concern in infertility has increased recently due to the decrease in the reproduction capability of couples worldwide [1]. Male reproductive defects contribute to nearly $50 \%$ of all infertility issues. Male infertility has been attributed to deteriorated sperm number or function [2]. Sperm dysfunction has been linked to varicocele, cryptorchidism, hypogonadism, testicular cancer, urogenital tract infections, systemic diseases and genetic factors [3]. However, in nearly $25 \%$ of male infertility cases,

\footnotetext{
*Correspondence: ayad.palani@garmian.edu.krd

${ }^{1}$ Research Center, University of Garmian, Kalar, Iraq

Full list of author information is available at the end of the article
}

men have demonstrated normal sperm parameters but still unable to father a child, which is termed unexplained infertility [4].

Oxidative stress (OS) has been involved in unexplained male infertility, and contemporary parameters have introduced OS as markers in the identification of potential causes of male infertility. OS is a state of imbalance between production and removal of reactive oxygen species (ROS) [5]. Antioxidants are compounds capable of scavenging and inhibiting the formation of ROS [6]. ROS have critical rule in male reproductive function, and these active molecules are required in small quantities for normal sperm function [7], while high quantities could decrease semen quality and impair sperm 
fertilization capability [8]. Hence, antioxidants are critical to inactivate ROS continuously to maintain only the small amount required to normal sperm function [9].

Recently, there is growing evidence suggesting that oxidative stress is involved in many aspects of male infertility and antioxidants counteract the adverse effects of OS on male fertility potential [10]. Antioxidants in seminal fluid can be classified into two forms: enzymatic and nonenzymatic antioxidants. Low seminal plasma antioxidants have been found to be implicated in reducing sperm concentration, motility and morphology [11]. Total antioxidant capacity (TAC) has shown to be strongly correlated with semen quality [12]. Similarly, glutathione has a role in ROS detoxification and protection of sperm plasma membrane [13]. Uric acid protects sperm viability and enhances sperm motility through stabilizing of ascorbic acid and scavenging of ROS [14, 15]. Albumin protects the spermatozoa against lipid peroxidation and prevents loss of sperm motility [16]. Malondialdehyde (MDA) is an index of lipid peroxidation; it is negatively correlated with sperm count, motility and morphology [17].

The aim of this study is to investigate the role oxidative stress in the pathogenesis of unexplained male infertility through estimation of semen and serum levels of TAC, glutathione, MDA, uric acid and albumin in normozoospermic infertile men.

\section{Methods}

This study was approved by ethical committee of university of Garmian (EC-G/191). Semen and serum samples were obtained from normozoospermic men after they were asked to fill consent form, during the period (March to November, 2017). A total of 64 subjects were included in this study, 42 infertile men with mean age $(32.5 \pm 6.3)$ years, and 22 fertile men with mean age $(33.9 \pm 7.2)$ years. Exclusion criteria for selection of subjects were: (1) smoking, (2) alcohol consumption, (3) abnormal BMI, (4) taking antioxidant medications, (5) etiologies related to physiological abnormality, such as varicocele, obstruction or small testicular volume and (6) having partner with low reproductive potential. Infertile men were attended to fertility clinics at Kalar city for infertility evaluation. Fertile men who had at least 1 child were enrolled in the study. Based on basic semen analysis results of infertile men, only participants with normal seminal fluid analysis were included in this study.

Semen collection and analysis Semen samples were obtained from patients and control group either by masturbation in the clinic or at home (delivered within $30 \mathrm{~min}$ ) after 3-4 days of sexual abstinence. Basic semen analyses were performed after liquefaction following the WHO 2010 manual. Samples from different laboratories were reanalyzed at our laboratory and performed by the same investigator after exactly $60 \mathrm{~min}$ for the sake of data consistency.

Blood collection Along with semen sample collection, subjects were asked for venous blood sample. Blood samples were collected by venipuncture into plastic vacutainer tube, and then, sera were separated and transferred to different tubes and stored in deep freeze $\left(-45^{\circ} \mathrm{C}\right)$ for further analyses of oxidative stress markers.

Estimation of total antioxidant capacity TAC was estimated using a colorimetric kit (Cell Biolabs, San Diego, CA, USA). The assay was based on the reduction of copper (II) to copper (I) by antioxidants and formation of a brown color with maximum absorbance at $490 \mathrm{~nm}$. The absorbance of the samples was compared with a known uric acid standard curve. Absorbance values were proportional to the samples total antioxidant capacity.

Estimation of glutathione Glutathione was estimated using a method by Moron et al. The solution was incubated with $0.1 \mathrm{ml}(10 \mathrm{mM}$ DTNB) and $10 \mu \mathrm{l}$ of $10 \%$ TCA at room temperature for $5 \mathrm{~min}$. The optical density of the yellow color complex was measured at $412 \mathrm{~nm}$. The concentration was calculated by plotting on glutathione standard curve [18].

Estimation of uric acid Uric acid was estimated using a colorimetric kit (Biolabo, Maizy, France). $20 \mu \mathrm{l}$ of seminal plasma was added to $1.0 \mathrm{ml}$ of uric acid reagent and incubated at $37^{\circ}$ for $5 \mathrm{~min}$. The developed color was read at $520 \mathrm{~nm}$. Concentrations were calculated and expressed as $\mathrm{mmol} / \mathrm{ml}$.

Estimation of albumin Albumin was estimated using a colorimetric kit (Biolabo, Maizy, France) at $\mathrm{pH}$ (4.3). Albumin binds to the BCG to form a BCG-albumin complex. The resulted green complex absorbed light at $630 \mathrm{~nm}$, and it was directly proportional to the concentration of albumin in the sample.

Estimation of malondialdehyde (MDA) MDA was measured by the reaction with thiobarbituric acid (TBA). Sample was added to $0.9 \mathrm{ml} \mathrm{D.W}$ and $0.5 \mathrm{ml}$ of TBA reagent. The mixture was heated for $1 \mathrm{~h}$ in a boiling water bath. The developed pink color was read at $532 \mathrm{~nm}$. The MDA level was expressed as (nmol/mL) [19].

\subsection{Statistical analysis}

MedCalc for Windows, version 14.8.1 (MedCalc Software, Ostend, Belgium) was used for statistical analysis. 
Semen parameters were expressed as Mean \pm SD. Parameters were tested for normality using KolmogorovSmirnov test. Significant difference between infertile and fertile groups was calculated using Mann-Whitney independent sample test. Differences at $p$ value $\leq 0.05$ were considered as significant.

\section{Results}

Semen basic parameters of infertile and fertile men are presented in Table 1 . No significant differences were observed in semen volume and sperm concentration, total count, motility, progressive motility, non-progressive motility and morphology.

TAC was significantly $(p<0.0001)$ lower in seminal plasma of infertile men, while no significant differences in glutathione, uric acid, albumin and MDA levels were observed (Fig. 1). In the comparison of oxidative stress parameters in blood serum, serum TAC level in normozoospermic infertile men was significantly $(p<0.01)$ lower than of fertile men, and no significant differences in glutathione, uric acid, albumin and MDA levels were found (Fig. 2).

\section{Discussion}

Normal semen parameters do not guarantee fertility; many factors may be related to male infertility rather than sperm count, motility and morphology which are not included in basic semen analysis [20]. However, new biochemical parameters have been studied widely over the last decades including oxidative stress to help in identifying the underlying cause of unexplained male infertility $[21,22]$.

Recently, growing evidences have indicated the role of TAC in male infertility. Decreasing seminal plasma antioxidant status may have a significant role in the etiology of sperm abnormality [23]. In our study, TAC was lower in the seminal plasma of infertile men (Fig. 1); this finding agrees with previously reported results $[24,25]$. The negative correlation between ROS and antioxidants has been confirmed; low seminal antioxidant potential allows
ROS overproduction in infertile men [26]. The exposure to ROS is extremely destructive to sperm plasma membrane [12]. Sperm-oocyte fusion is a membrane-dependent processes; oxidation of sperm membrane impairs the penetration ability of the spermatozoa and causes infertility in normozoospermic men [27].

Along with seminal antioxidants assessment, serum TAC determination has been recently proposed as a valuable marker to evaluate the sperm quality [28]. However, serum oxidative stress marker in relation to male infertility is less investigated and poorly understood [29]. Serum levels of TAC were significantly different $(p<0.01)$ between infertile and fertile men (Fig. 2). This finding is in agreement with the results of Benedetti et al. [29], and with our previous report [30], both were reported a decrease in serum TAC in infertile compared to fertile men. In contrast, our results disagree with Eroglu et al. [31]; they reported no significant difference in serum TAC between normozoospermic infertile and fertile men. Glutathione showed to be influencing sperm motility by way of prevention of lipid peroxidation of sperm cell membrane [32]. However, no significant difference in semen and serum glutathione level was found between the different groups. These results agree with previous reports by Guz et al. [33] and Feng et al. [34]; they reported no significant differences in semen and serum uric acid concentration between fertile and infertile men $[33,34]$. However, in a disagreement, Bhardwaj et al. [35] reported a significant decrease in semen glutathione level in infertile men, and Thiele et al. [14] reported significant decrease in uric acid concentration in normozoospermic infertile men comparing to fertile men.

Few studies have investigated the possible relationship between seminal plasma albumin and sperm quality; a weak positive correlation between albumin and sperm concentration was reported [36]. In the present study, no significant difference in albumin concentration was found. MDA has related to glutathione concentration, and both have shown to influence sperm quality [37]. MDA level has been reported

Table 1 Basic semen parameters of infertile and fertile men

\begin{tabular}{lccc}
\hline Semen characteristics & Infertile men $(\boldsymbol{n = 4 6 )}$ & Fertile men $(\boldsymbol{n = 2 1 )}$ & $\boldsymbol{p}$ value \\
\hline Semen volume $(\mathrm{ml})$ & $3.9 \pm 1.4$ & $4.2 \pm 1.8$ & $\mathrm{NS}$ \\
Sperm concentration $\left(10^{6} / \mathrm{ml}\right)$ & $67.5 \pm 46.3$ & $63.0 \pm 28.3$ & $\mathrm{NS}$ \\
Sperm total count $\left(10^{6} /\right.$ ejaculate) & $249.9 \pm 193.2$ & $284.4 \pm 193.4$ & $\mathrm{NS}$ \\
Progressive motility (\%) & $55.2 \pm 8.9$ & $56.9 \pm 11.5$ & $\mathrm{NS}$ \\
Non-progressive motility (\%) & $21.5 \pm 14.1$ & $18.0 \pm 14.3$ & $\mathrm{NS}$ \\
Total motility (\%) & $75.6 \pm 9.0$ & $75.3 \pm 9.4$ & $\mathrm{NS}$ \\
Normal morphology $(\%)$ & $15.3 \pm 10.7$ & $16.0 \pm 3.4$ & $\mathrm{NS}$ \\
\hline
\end{tabular}

NS No significant difference $(p \leq 0.05)$ 


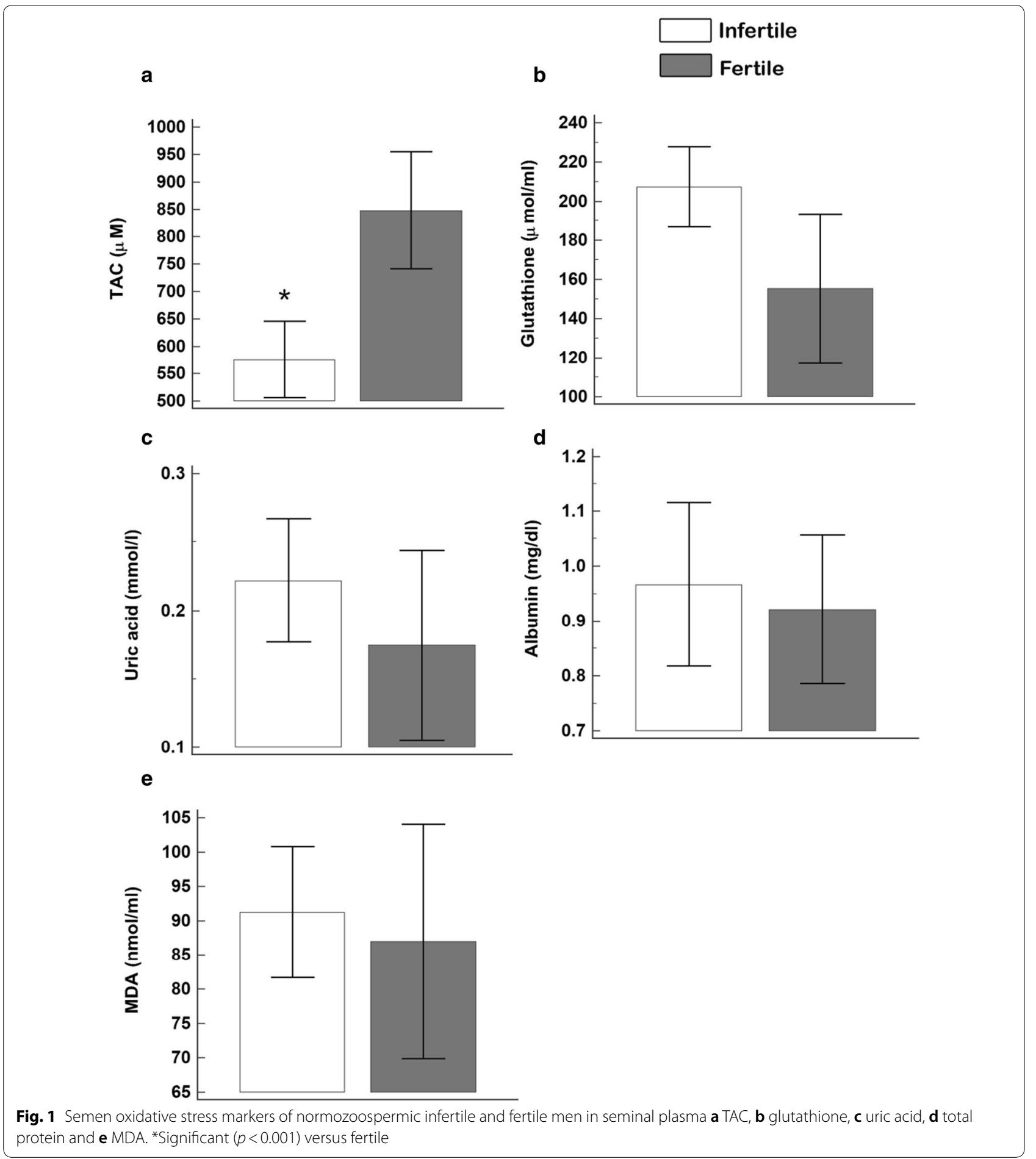

to negatively correlate with sperm concentration and motility [38]; MDA levels do not significantly differ between the studied infertile and fertile groups. However, it was previously reported that MDA has negatively correlated with sperm quality and significantly higher in seminal plasma of infertile men [39, 40]. One explanation for the discrepancy of the findings of the aforementioned studies is the heterogeneity of studies in selection of patients, methodologies, methods used to assess OS as well as genetic and racial factors. Our 
a
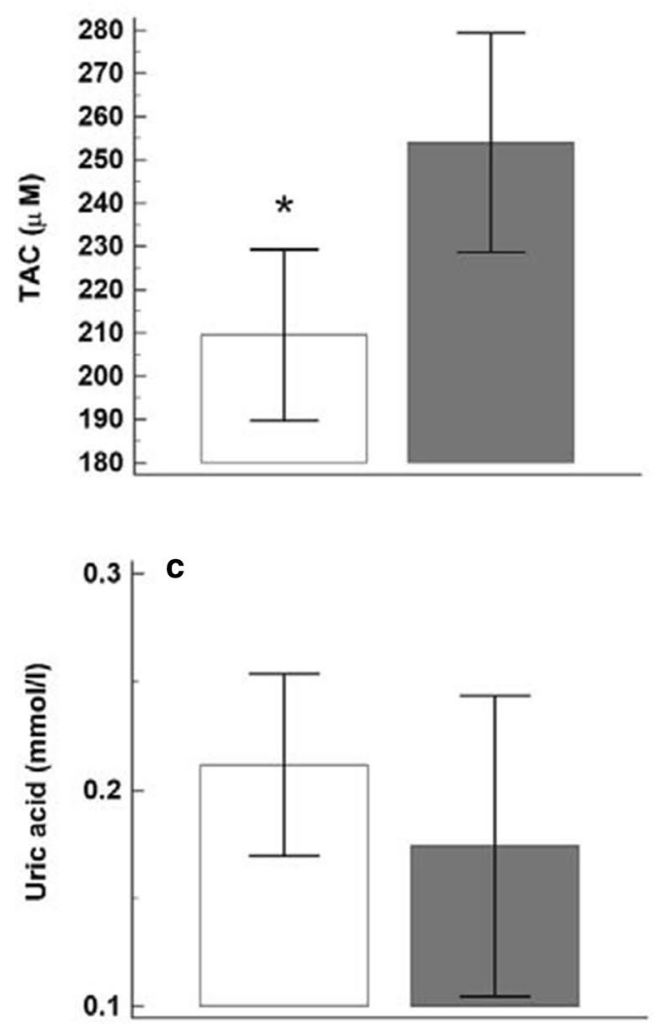

d

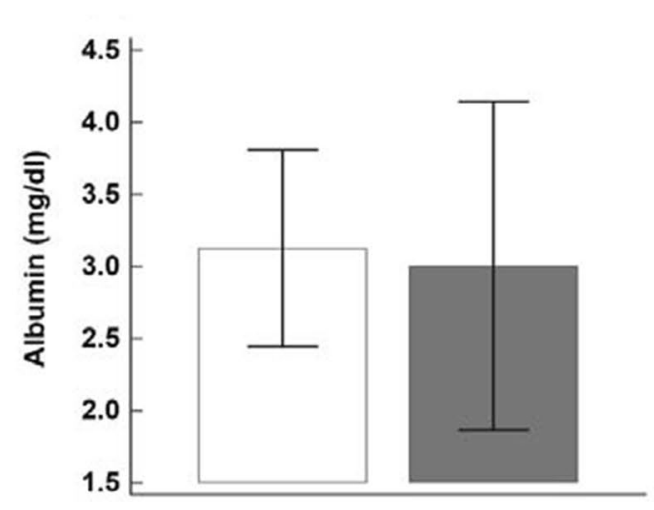

b

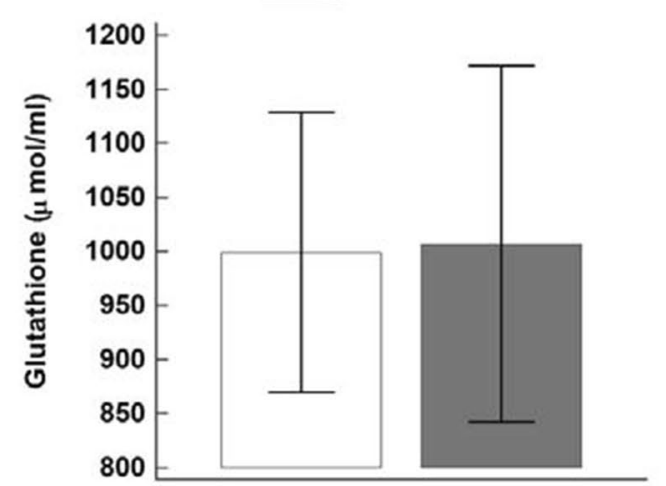

Infertile

$\square$ Fertile

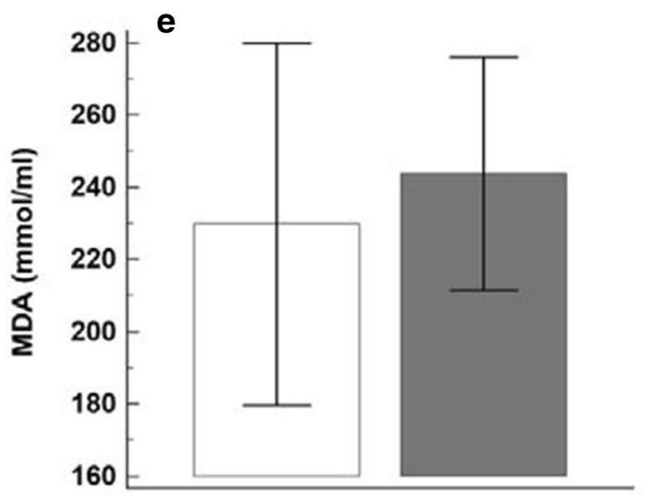

Fig. 2 Serum oxidative stress markers of Idiopathic infertile and fertile men in seminal plasma a TAC, b glutathione, $\mathbf{c}$ uric acid, $\mathbf{d}$ total protein and $\mathbf{e}$ MDA. *Significant $(p<0.01)$ versus fertile

study has some limitations which include the small number of participants and the lack of long term follow up, so further large-scale prospective studies are warranted to consolidate the evidence presented in this study.

\section{Conclusion}

Our study demonstrates that total antioxidant capacity (TAC) affects male fecundity. TAC level induces oxidative stress and consequently declines sperm fertilization capability even in men with normal basic sperm parameters. Estimation of TAC can be used as an investigative 
marker in the diagnosis of male infertility. The beneficial usage of antioxidants should be considered in the treatment of oxidative stress-induced male infertility. Further clinical trials are needed to investigate the therapeutic effect of antioxidant supplementation in the treatment of male infertility.

\section{Abbreviations}

OS: Oxidative stress; TAC: Total antioxidant capacity; ROS: Reactive oxygen species; MDA: Malondialdehyde; TBA: Thiobarbituric acid; BCG: Bromocresol green DTNB: 5,5'-Dithiobis-(2-nitrobenzoic acid); BMI: Body mass index; DW: Distilled water; WHO: World Health Organization.

\section{Acknowledgements}

The authors are grateful to the staff of Zanko medical laboratory at Kalar, Sulaymaniyah, Iraq, for helping and providing facilities in sample collection.

\section{Authors' contributions}

AP has done the research and wrote the article, and AA has improved the scientific writing of the manuscript. Both authors read and approved the manuscript.

\section{Funding}

Not applicable.

\section{Availability of data and materials}

The datasets used and/or analyzed during the current study are available from the corresponding author on reasonable request.

\section{Ethics approval and consent to participate}

This study was approved by the Institutional Review Board of the University of Garmian (Permit No.: IRB-G: 37). All patients were participated after filling consent form written in their native language.

\section{Consent for publication}

Not applicable.

\section{Competing interests}

Authors declare that there are no competing interests.

\section{Author details}

${ }^{1}$ Research Center, University of Garmian, Kalar, Iraq. ${ }^{2}$ Department of Pathological Analysis, College of Science, University of Sumer, Thi-Qar, Iraq.

Received: 2 April 2020 Accepted: 10 August 2020

Published online: 31 October 2020

\section{References}

1. Palani A et al (2020) Geographical differences in semen characteristics: comparing semen parameters of infertile men of the United States and Iraq. Andrologia 52:e13519

2. Alahmar AT (2018) The effects of oral antioxidants on the semen of men with idiopathic oligoasthenoteratozoospermia. Clin Exp Reprod Med 45:57-66

3. Selvam MKP, Sengupta P, Agarwal A (2020) Sperm DNA fragmentation and male infertility. In: Genetics of male infertility. Springer, Cham

4. Khalil AA, Hussien HM, Sarhan EM (2012) Oxidative stress induces idiopathic infertility in Egyptian males. Afr J Biotechnol 11:1516-1522

5. Özdamar A, Soylu A, Culha M et al (2004) Testicular oxidative stress. Urol Int 73:343-347

6. Sikka SC, Rajasekaran M, Hellstrom WJ (1995) Role of oxidative stress and antioxidants in male infertility. J Androl 16:464-468

7. Hsieh Y-Y, Chang C-C, Lin C-S (2006) Seminal malondialdehyde concentration but not glutathione peroxidase activity is negatively correlated with seminal concentration and motility. Int J Biol Sci 2:23
8. Kefer JC, Agarwal A, Sabanegh E (2009) Role of antioxidants in the treatment of male infertility. Int J Urol 16:449-457

9. Agarwal A, Saleh RA (2002) Role of oxidants in male infertility: rationale, significance, and treatment. Urol Clin North Am 29:817-828

10. Alahmar AT (2019) Role of oxidative stress in male infertility: an updated review. J Hum Reprod Sci 12:4

11. Roychoudhury S, Sharma R, Sikka S et al (2016) Diagnostic application of total antioxidant capacity in seminal plasma to assess oxidative stress in male factor infertility. J Assist Reprod Gen 33:627-635

12. Pahune PP, Choudhari AR, Muley PA (2013) The total antioxidant power of semen and its correlation with the fertility potential of human male subjects. J Clin Diagn Res 7:991-995

13. Ochseedorf FR, Buhl R, Bästlein A et al (1998) Glutathione le spermatozoa and seminal plasma of infertile men. Hum Reprod 13:353-359

14. Thiele J, Freisleben H, Fuchs $J$ et al (1995) Ascorbic acid and urate in human seminal plasma: determination and interrelationships with chemiluminescence in washed semen. Hum Reprod 10:110-115

15. Banihani SA (2018) Role of uric acid in semen. Biomolecules 2018(8):65

16. Alvarez JG, Storey BT (1983) Taurine, hypotaurine, epinephrine and albumin inhibit lipid peroxidation in rabbit spermatozoa and protect against loss of motility. Biol Reprod 29:548-555

17. Colagar AH, Pouramir M, Marzony ET et al (2009) Relationship between seminal malondialdehyde levels and sperm quality in fertile and infertile men. Braz Arch Biol Technol 52:1387-1392

18. Moron MS, Depierre JW, Mannervik B (1979) Levels of glutathione, glutathione reductase and glutathione S-transferase activities in rat lung and liver. Biochim Biophys Acta (BBA)-General Subjects 582:67-78

19. Rao B, Soufir J, Martin M et al (1989) Lipid peroxidation in human spermatozoa as relatd to midpiece abnormalities and motility. Gamete Res 24:127-134

20. Pasqualotto F, Sharma R, Kobayashi $\mathrm{H}$ et al (2001) Oxidative stress in normospermic men undergoing infertility evaluation. J Androl 22:316-322

21. Cayli S, Jakab A, Ovari L et al (2003) Biochemical markers of sperm function: male fertility and sperm selection for ICSI. Reprod Biomed Online 7:462-468

22. Diamandis EP, Arnett WP, Foussias G et al (1999) Seminal plasma biochemical markers and their association with semen analysis findings. Urology 53:596-603

23. Khosrowbeygi A, Zarghami N, Deldar Y (2012) Correlation between sperm quality parameters and seminal plasma antioxidants status. Int J Reprod Biomed 2:58-64

24. Palani AF, Asdallh NS (2019) Effects of Low Seminal Plasma Antioxidant Potential on Semen Quality and Male Fertility. Passer J 1:4-8

25. Subramanian $V$ et al (2018) Seminal reactive oxygen species and total antioxidant capacity: correlations with sperm parameters and impact on male infertility. Clin Exp Reprod Med 45:88

26. Agarwal A, Sengupta P (2020) Oxidative stress and its association with male infertility. In: Male Infertility. Springer, Cham

27. Aitken RJ (2006) Sperm function tests and fertility. Int J Androl 29:69-75 (discussion 105-108)

28. Shamsi M, Venkatesh S, Kumar R et al (2010) Antioxidant levels in blood and seminal plasma and their impact on sperm parameters in infertile men. Indian J Biochem Biophys 47:38-43

29. Benedetti S, Tagliamonte MC, Catalani S et al (2012) Differences in blood and semen oxidative status in fertile and infertile men, and their relationship with sperm quality. Reprod Biomed Online 25:300-306

30. Palani AF (2018) Effect of serum antioxidant levels on sperm function in infertile male. Middle East Fertil Soc J 23(1):19-22

31. Eroglu M et al (2014) Blood serum and seminal plasma selenium, total antioxidant capacity and coenzyme $\mathrm{Q} 10$ levels in relation to semen parameters in men with idiopathic infertility. Biol Trace Elem Res 159:46-51

32. Moslemi MK, Tavanbakhsh S (2011) Selenium-vitamin E supplementation in infertile men: effects on semen parameters and pregnancy rate. I Int J Gen Med 4:99

33. Guz J, Gackowski D, Foksinski M et al (2013) Comparison of oxidative stress/DNA damage in semen and blood of fertile and infertile men. PLoS ONE 8:e68490

34. Feng RX et al (2015) A pilot comparative study of 26 biochemical markers in seminal plasma and serum in infertile men. Biomed Res Int. https://doi. org/10.1155/2015/805328 
35. Bhardwaj A, Verma A, Majumdar S et al (2000) Status of vitamin E and reduced glutathione in semen of oligozoospermic and azoospermic patients. Asian J Androl 2:225-228

36. Elzanaty S, Erenpreiss J, Becker C (2007) Seminal plasma albumin: origin and relation to the male reproductive parameters. Andrologia 39:60-65

37. Walczak-Jedrzejowska R, Wolski JK, Slowikowska-Hilczer J (2013) The role of oxidative stress and antioxidants in male fertility. Cent European J Urol 66:60

38. Collodel G, Moretti E, Micheli L et al (2015) Semen characteristics and malondialdehyde levels in men with different reproductive problems. Andrology 3:280-286
39. Fafula RV, Onufrovych OK, Vorobets DZ et al (2017) Glutathione antioxidant protection system in ejaculated spermatozoa of infertile men with different forms of pathospermia. Біологічні студії 11:17-24

40. Dorostghoal $\mathrm{M}$ et al (2017) Oxidative stress status and sperm DNA fragmentation in fertile and infertile men. Andrologia 49:e12762

\section{Publisher's Note}

Springer Nature remains neutral with regard to jurisdictional claims in published maps and institutional affiliations.

\section{Submit your manuscript to a SpringerOpen ${ }^{\circ}$ journal and benefit from:}

- Convenient online submission

- Rigorous peer review

- Open access: articles freely available online

- High visibility within the field

- Retaining the copyright to your article

Submit your next manuscript at $\boldsymbol{\nabla}$ springeropen.com 